\title{
Induction of apoptosis by obovatol as a novel therapeutic strategy for acute myeloid leukemia
}

\author{
HYENG-SOO KIM*, GA YOUNG LIM*, JUNMO HWANG, ZAE YOUNG RYOO, TAE-LIN HUH and SANGGYU LEE
}

\author{
School of Life Science, BK21 plus KNU Creative BioResearch Group, \\ Kyungpook National University, Daegu 702-701, Republic of Korea
}

Received June 16, 2014; Accepted September 30, 2014

DOI: 10.3892/ijmm.2014.1957

\begin{abstract}
Obovatol, a compound isolated from the bark cortex of Magnolia officinalis (cortex Magnoliae officinalis; M. officinalis), has been studied for use in the treatment of solid cancers. However, the mechanisms of action and the effects of obovatol against acute myeloid leukemia (AML) remain unclear and require further investigation. Therefore, this study was conducted using a human AML cell line (MM6). Obovatol increased pro-apoptotic (Bax) and decreased antiapoptotic (Bcl-2) protein expression, resulting in caspase-3 and caspase- 9 activation measured by caspase-Glo 3/7 assay. Furthermore, obovatol activated the mitogen-activated protein kinase (MAPK) signaling pathway [c-Jun N-terminal kinase (JNK), extracellular signal-regulated kinase (ERK) and p38] and inhibited the activation of the nuclear factor- $\mathrm{\kappa B}(\mathrm{NF}-\mathrm{\kappa B})$ signaling pathway analyzed by western blot analysis. Taken together, these findings provide evidence that obovatol inhibits cell proliferation in AML and induces apoptosis through the activation of the MAPK pathway in addition to the intrinsic apoptotic pathway. In addition, obovatol suppressed the expression of mixed-lineage leukemia (MLL) target genes by inhibiting the activation of the NF- $\mathrm{kB}$ pathway. Therefore, these results suggest that obovatol may have potential for use in the treatment of leukemia.
\end{abstract}

\section{Introduction}

Acute myeloid leukemia (AML) is characterized by the rapid growth of abnormal white blood cells that accumulate in the bone marrow and interfere with the production of normal blood cells. With the increasing understanding of its pathogen-

Correspondence to: Professor Sanggyu Lee, School of Life Science, BK21 plus KNU Creative BioResearch Group, Kyungpook National University, 1370 Sankyuk-dong, Buk-ku, Daegu 702-701, Republic of Korea

E-mail: slee@knu.ac.kr

*Contributed equally

Key words: obovatol, acute myeloid leukemia, apoptosis, mitogenactivated protein kinase, nuclear factor- $\kappa$ B, HOXA9 esis, cytotoxic chemotherapy with or without follow-up with hematopoietic cell transplantation is the primary treatment for AML. Although much effort has been made regarding AML treatment, the prognosis remains dismal. The development of novel therapies is thus highly desirable (1).

The bark of Magnolia officinalis (cortex Magnoliae officinalis; M. officinalis) has been widely used as a folk remedy for gastrointestinal disorders, cough, anxiety and allergies. Several compounds have been isolated from $M$. officinalis including magnolol, honokiol and obovatol $(2,3)$. Magnolol, a low molecular weight lignan $(4,5)$, has been shown to possess anti-platelet aggregation, anxiolytic, anti-fungal, anti-bacterial $(3,6,7)$, anti-viral, anti-carcinogenic (8), and anti-metastatic properties (9). It is also worth noting that honokiol has demonstrated anti-angiogenic, anti-invasive and anti-proliferate effects in a variety of cancer cells, including squamous cell lung cancer (10), leukemia (11-13) and multiple myeloma (14). Obovatol is the major biphenolic component of Magnolia obovata (M. obovata) leaves and is known to have anti-inflammatory and anti-tumor effects through the inhibition of nuclear factor- $\kappa \mathrm{B}(\mathrm{NF}-\kappa \mathrm{B})(15)$.

Previous studies have reported that obovatol inhibits cell growth through the induction of apoptotic cell death in solid cancers by blocking the NF- $\mathrm{KB}$ or mammalian target of rapamycin (mTOR) signaling pathways. However, to the best of our knowledge, the effects of obovatol in leukemia have not yet been reported. Thus, the aim of this study was to investigate the underlying mechanisms of action of obovatol using a leukemia cell line and to explore the possibility of its use as a therapeutic agent in the treatment of leukemia.

\section{Materials and methods}

Cell culture and compounds. Human AML cells, such as Jurkat, MM6, THP-1 and U937 cells (all purchased from ATCC, Manassas, VA, USA) were maintained in RPMI-1640 medium supplemented with $10 \%$ fetal bovine serum and $1 \%$ penicillin/ streptomycin (Gibco-BRL, Grand Island, NY, USA). The cells were incubated at $37^{\circ} \mathrm{C}$ in a humidified atmosphere of $5 \% \mathrm{CO}_{2}$. Solutions $(100 \mathrm{mM})$ of obovatol were prepared with dimethyl sulfoxide (DMSO), stored at $-20^{\circ} \mathrm{C}$, and then diluted as needed in the cell culture medium. Obovatol (purity $>98 \%$ ) was kindly provided by the Korea Research Institute of Bioscience and Biotechnology (Daejeon, Korea). 
Assessment of cell viability and caspase-3/caspase-7 expression . The cells were seeded at a density of $3 \times 10^{4}$ cells/well in a 96-well microtiter plate. After $24 \mathrm{~h}$, the medium in the wells was replaced with fresh complete medium containing various concentrations of obovatol $(0-80 \mu \mathrm{M})$ in $0.1 \%$ DMSO for $48 \mathrm{~h}$, or the medium was replaced with fresh complete medium containing $60 \mu \mathrm{M}$ of obovatol and maintained for different periods of time (3-48 h). Cell viability was determined using the CCK-8 kit (Dojindo, Kumamoto, Japan). The cells $\left(1 \times 10^{4}\right)$ were seeded in 96-well plates and then treated with $60 \mu \mathrm{M}$ obovatol for $1,4,7$ and $10 \mathrm{~h}$, and caspase activity was determined with the Caspase-Glo 3/7 assay (Promega, Madison, WI, USA), according to the manufacturer's instructions. The intensity of luminescence in a plate-reading luminometer was measured (Perkin Elmer Victor3 multilabel counter; PerkinElmer, Inc., Waltham, MA, USA).

Flow cytometric analysis. For cell cycle analysis, the cells were treated with $60 \mu \mathrm{M}$ of obovatol for $24-48 \mathrm{~h}$. The cells were then washed with phosphate-buffered saline (PBS) and fixed with icecold $70 \%$ ethanol for $30 \mathrm{~min}$. The fixed cells were resuspended in PBS (100 $\mu \mathrm{l} / 1 \times 10^{5}$ cells) and treated with $100 \mu \mathrm{g} / \mathrm{ml}$ of RNase A and stained with $100 \mu \mathrm{g} / \mathrm{ml}$ of propidium iodide (PI) for $40 \mathrm{~min}$. The DNA content was analyzed on a FACScalibur flow cytometer (BD Biosciences, Bedford, MA, USA). To analyze apoptosis, the cells were collected at 24-48 h following treatment with obovatol, washed with PBS, and stained with Alexa Fluor $^{\circledR}$ 488-conjugated Annexin V and PI (Invitrogen, Carlsbad, CA, USA). The stained cells were analyzed by flow cytometry.

Reverse transcription-quantitative polymerase chain reaction (RT-qPCR). The cells were treated with obovatol for $24 \mathrm{~h}$ and total RNA was extracted using the RNeasy Mini kit (Qiagen, Valencia, CA, USA) according to the instructionsof the manufacturer. cDNA synthesis was performed using M-MLV Reverse Transcriptase according to the insructions of the manufacturer (Invitrogen). RT-qPCR was performed with StepOnePlus (Applied Biosystems, Foster City, CA) using SYBR Premix Ex Taq (Takara Bio, Inc., Shiga, Japan) with the following primers: ACTB sense, 5'-TGAGATGCGTTGTT ACAGGAAGTC-3' and antisense, 5'-GACTGGGCCATT CTCCTTAGAGA-3'; Bak sense, 5'-CAGCACCCTAAG AGATGGGACTA-3' and antisense, 5'-CCTGCTCC TGGGACACATG-3'; Bax, 5'-GCCGCCGTGGACACA-3' and antisense, 5'-TTGCCGTCAGAAAACATGTCA-3'; Bim, sense, 5'-TTCGGGTCCTGGTATTTCCA-3' and antisense, 5'-GGCATCAAACACACACTTCATCA-3'; Puma sense, 5'-GGGCCCAGACTGTGAATCCT-3' and antisense, 5'-CGTGCTCTCTCTAAACCTATGCAA-3'; Bcl-2 sense, 5'-TGGTACGACCTTTAGATTCCAGAGA-3' and antisense, 5'-CCCATTAGACATATCCAGCTTGAA-3').

Western blot analysis. The cells were cultured with obovatol for the indicated periods of time. Following treatment, the cells were harvested, washed twice with cold PBS and lysed on ice. Western blot analysis with antibodies to Akt, p38, phosphoryalted (p-)p38, extracellular signal-regulated kinase (ERK), c-Jun N-terminal kinase (JNK), IKK $\beta$, I $\kappa \mathrm{B} \alpha, \mathrm{p}-\mathrm{I} \kappa \mathrm{B} \alpha(\mathrm{Ser} 32 / 36), \mathrm{p}-\mathrm{p} 65$ (Ser536), Bax, Bcl-2 (Cell Signaling Technology, Beverly, MA, USA), p-Akt (Thr308), p-JNK, p-ERK (Bioworld Technology, Saint Louis Park, MN, USA), homeobox A9 (HOXA9), p-retinoblastoma protein (Rb; Ser780) and $\beta$-actin (Santa Cruz Biotechnology, Inc., Santa Cruz, CA, USA) was performed.

Data analysis and statistics. Data are presented as the means \pm standard deviation (SD). All the experiments were performed a minimum of 3 times. Statistical analyses were performed using the unpaired sample two-tailed Student's t-test. A P-value < 0.05 was considered to indicate a statistically significant difference.

\section{Results}

Obovatol inhibits cell proliferation and cell cycle progression. To determine the biological activity of obovatol in leukemia cells, we investigated whether the obovatol inhibits the proliferation of leukemia cells (Jurkat, MM6, THP-1 and U937) following treatment with various concentrations of obovatol $(0-80 \mu \mathrm{M})$ for different periods of time $(3,6,12,24$ and $48 \mathrm{~h})$. DMSO was used as a negative control in most of the experiments. We measured the cytotoxicity of obovatol indirectly using the CCK- 8 assay. The viability of all the leukemia cells decreased in a concentration- and a time-dependent manner (Fig. 1A and B). In particular, the viability of the MM6 cells was affected the greatest by obovatol treatment at the same concentrations. To determine the effects of obovatol on the cell cycle, the cell cycle distribution was examined by FACS analysis. The treatment of MM6 cells with obovatol led to an accumulation of cells in the sub-G1 phase compared with the control cells, coupled with a concomitant decrease in the proportion of cells in the $\mathrm{S}$ and $\mathrm{G} 2 / \mathrm{M}$ phases in a timedependent manner (Fig. 1C). In addition, obovatol significantly decreased the phosphorylation of $\mathrm{Rb}$ in a time-dependent manner (Fig. 1D). Based on these results, we hypothesized that obovatol inhibits cell proliferation and cell cycle progression in either non- or mixed-lineage leukemia (MLL)-rearranged human leukemia cells.

Obovatol induces apoptosis through the caspase-dependent pathway. In order to further elucidate the effects of obovatolinduced growth inhibition, we investigated whether the increased accumulation of cells in the sub-G1 phase was due to apoptosis or necrosis. FACS analysis was carried out after staining the cells with Annexin V (FITC) and PI. Treatment with a concentration of $60 \mu \mathrm{M}$ of obovatol for 24 and $48 \mathrm{~h}$ increased the number of apoptotic cells (Fig. 2A). As Bcl-2 family proteins are key factors in controlling the mitochondrial-dependent apoptotic pathway (16), we further examined the expression levels of anti- and pro-apoptotic molecules in obovatol-treated and untreated MM6 cells. The increased transcriptional expression of Bak, Bax, Bim and Puma (proapoptotic proteins) and the decreased expression of $\mathrm{Bcl}-2$ (anti-apoptotic protein) was observed in the obovatol-treated MM6 cells after $24 \mathrm{~h}$ (Fig. 2B). The protein expression levels of Bax increased after $4 \mathrm{~h}$, while Bcl-2 expression decreased after $1 \mathrm{~h}$ in a concentration-dependent manner, but started to increase after $4 \mathrm{~h}$ of treatment with $60 \mu \mathrm{M}$ obovatol (Fig. 2C and D). Moreover, since the caspase-dependent pathway plays a pivotal role in apoptosis (17) and a previous study demonstrated that obovatol induced apoptosis by regulating the caspase-dependent pathway in prostate and colon cancer 

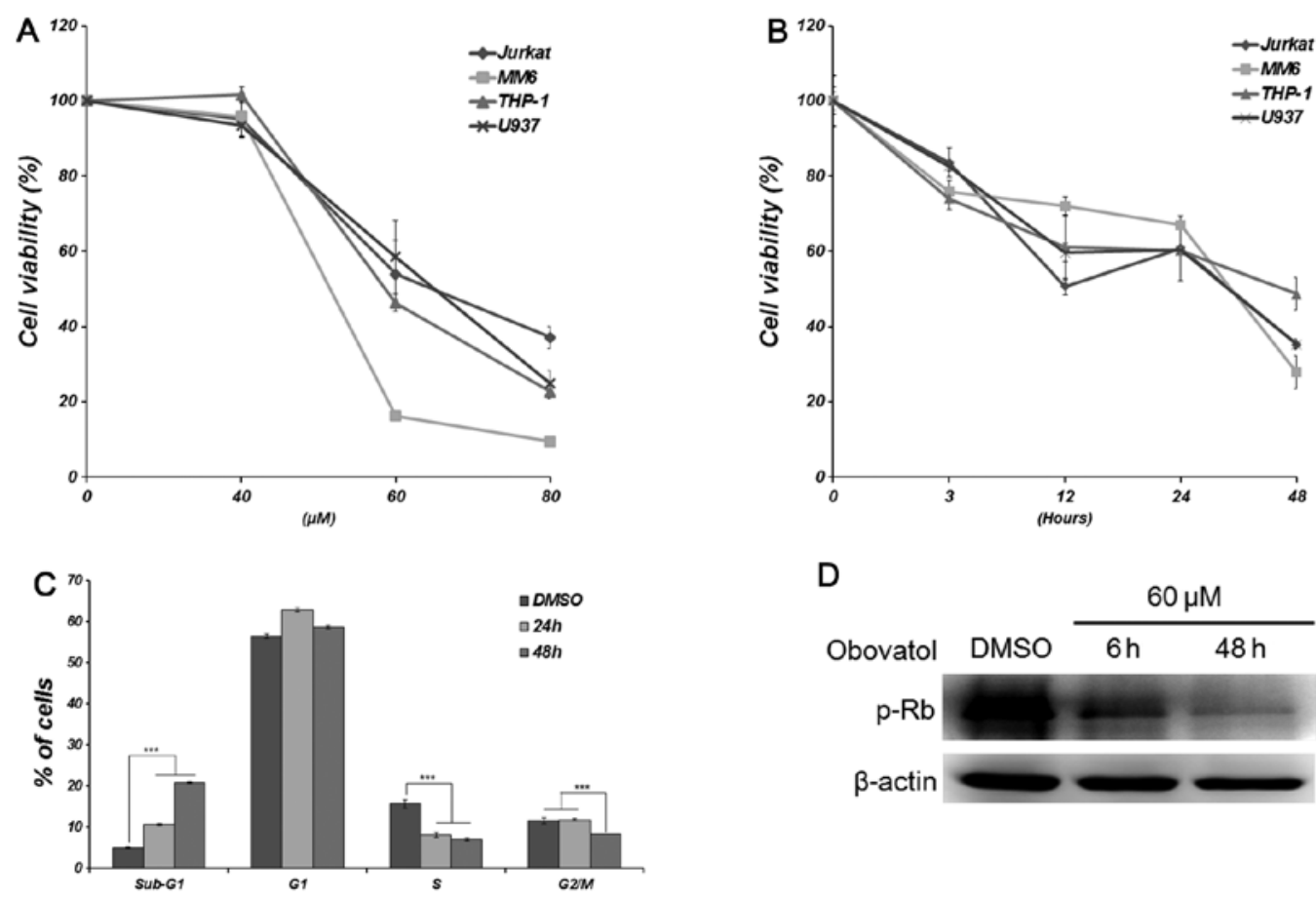

Figure 1. Effects of obovatol on growth of leukemia cells. Various leukemia cell lines were treated with obovatol $(40-80 \mu \mathrm{M})$ for $48 \mathrm{~h}$ or obovatol (60 $\mu \mathrm{M})$ for (3-48 h). The viability of the cells was detected by CCK-8 assay. Obovatol inhibited the growth of various leukemia cells in (A) a time- and (B) concentrationdependent manner. MM6 cells were harvested with $60 \mu \mathrm{M}$ obovatol for $24-48 \mathrm{~h}$, fixed, and stained with propidium iodide (PI). The stained cells were subjected to flow cytometric analysis to determine the distribution of cells. (C) Cell cycle distribution of MM6 cells in the absence or presence of obovatol for 24 and $48 \mathrm{~h}$. ${ }^{* * * * *} \mathrm{P}<0.001$ indicates statistical significance. Error bars indicate the mean \pm standard deviation (SD). (D) Effects of treatment with obovatol for 6 and $48 \mathrm{~h}$ on the expression of phosphorylated (p-)Rb.
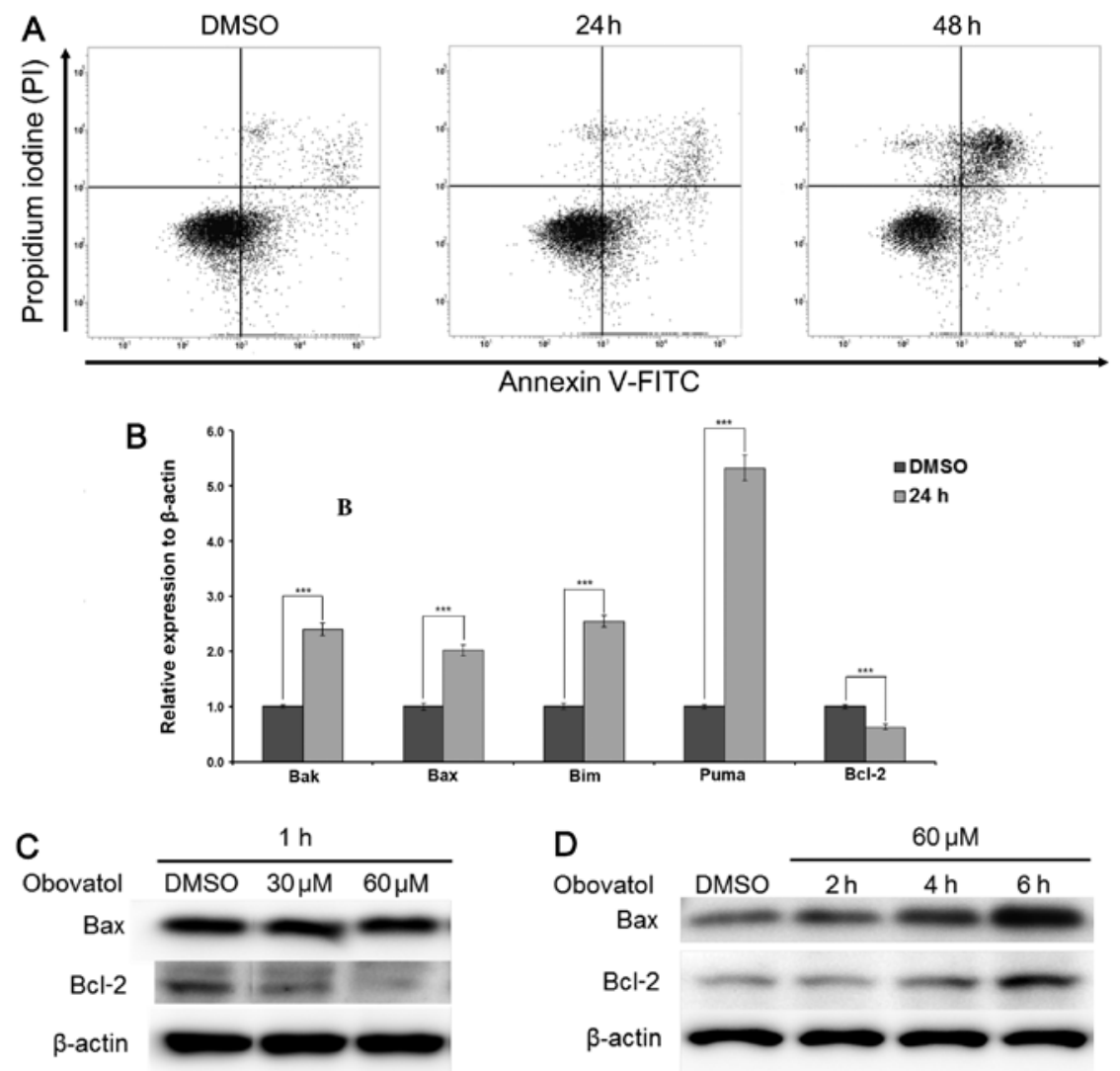

Figure 2. Induction of apoptosis by obovatol. MM6 cells were stained with Annexin V (FITC) and propidium iodide (PI) for 15 min following treatment with $60 \mu \mathrm{M}$ obovatol for 24 and $48 \mathrm{~h}$ and then analyzed by FACS. (A) The number of apoptotic cells increased over time. mRNA expression of Bak, Bax, Bim, Puma and Bcl-2 induced by $60 \mu \mathrm{M}$ obovatol for $24 \mathrm{~h}$. Total RNA was prepared from MM6 cells following treatment with $60 \mu \mathrm{M}$ obovatol for $24 \mathrm{~h}$ and the levels of Bak, Bax, Bim, Puma and Bcl-2 were assessed by RT-qPCR. (B) mRNA expression of pro- and anti-apoptotic genes. Protein expression levels of Bax and Bcl-2 (C) after $1 \mathrm{~h}$ and (D) after 2-6 h. ${ }^{* * *} \mathrm{P}<0.001$ indicates statistical significance. Error bars indicate the mean \pm standard deviation (SD). 
A

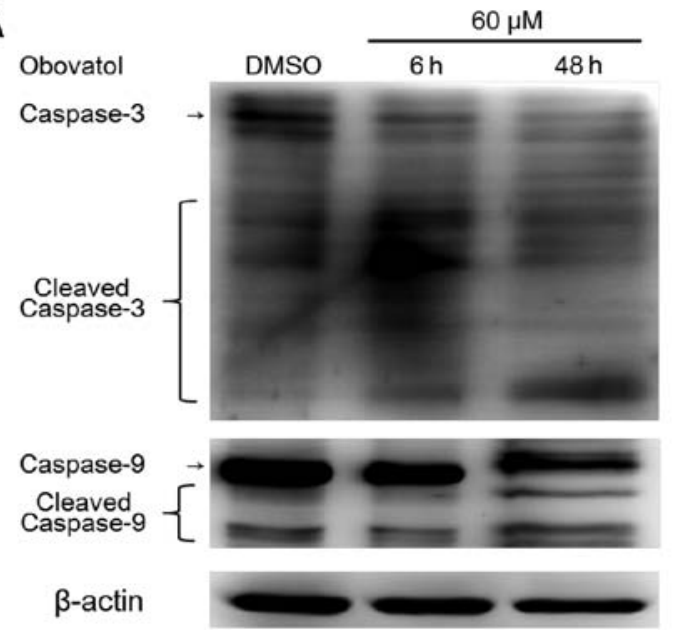

B

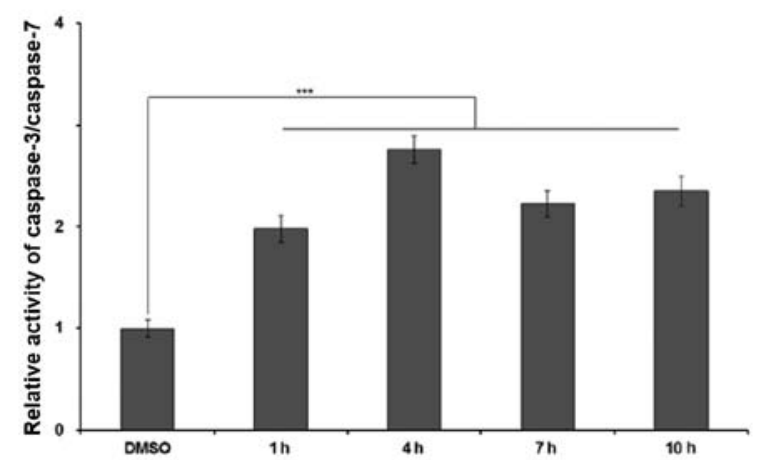

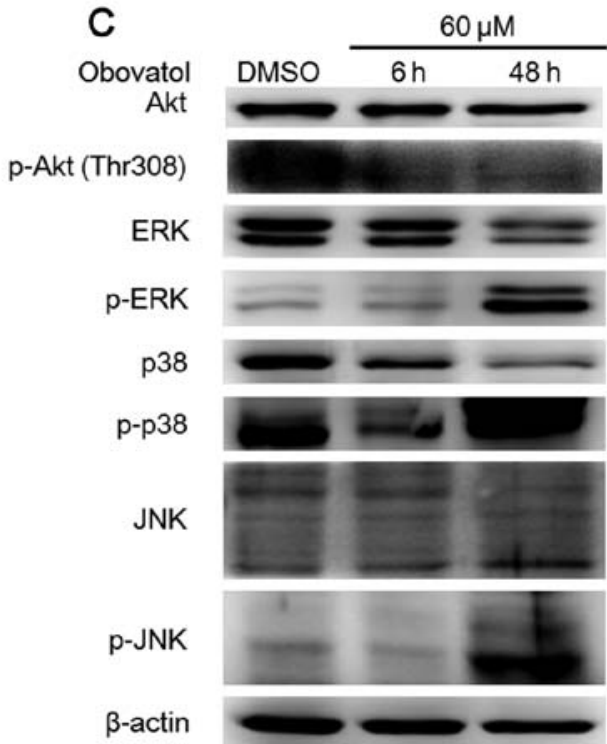

D

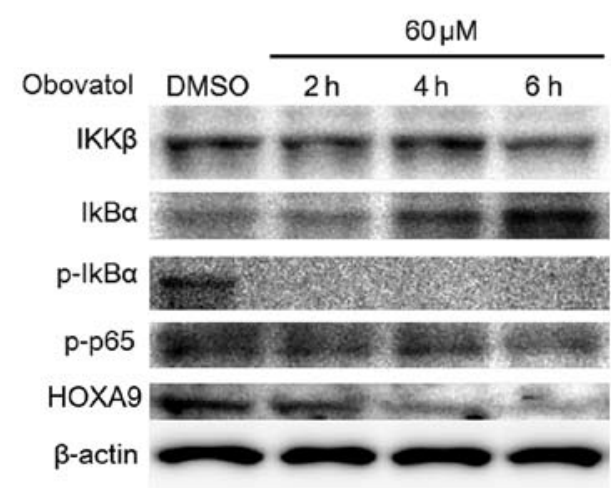

Figure 3. (A) Activation of caspase-3 and caspase- 9 induced by obovatol. (B) Relative expression of caspase- $3 /$ caspase- 7 in the absence or presence of $60 \mu \mathrm{M}$ obovatol for $1-10 \mathrm{~h} .{ }^{* * * *} \mathrm{P}<0.001$ indicates statistical significance. Error bars indicate the mean \pm standard deviation (SD). (C) Effects of obovatol on the expression of Akt and the MAPK signaling pathway. MM6 cells either untreated or treated with $60 \mu \mathrm{M}$ obovatol. Equal amounts of total protein were subjected to $10 \%$ SDSPAGE. Expression of total-Akt, extracellular signal-regulated kinase (ERK), c-Jun N-terminal kinase (JNK), phosphorylated (p-)p38, p-Akt, ERK, JNK and p38 was detected by western blot analysis using specific antibodies. (D) Effects of obovatol on NF- $\mathrm{KB}$ signaling pathway. MM6 cells were treated with dimethyl

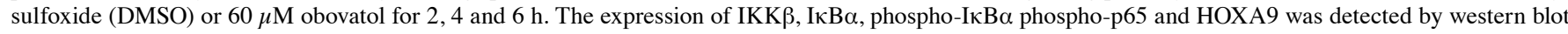
analysis. $\beta$-actin protein expression was used as an internal control.

cells (18), we examined the expression of proteins associated with caspase-related mitochondrial-dependent apoptosis in the obovatol-treated MM6 cells. As expected, the active fragments of caspase- 3 and caspase- 9 were observed following treatment with obovatol for 6-48 h (Fig. 3A). Additionally, there was a sustained increase in the relative activity of caspase-3/ caspase-7 after $1 \mathrm{~h}$ (Fig. 3B). These results collectively suggest that obovatol induces apoptosis rapidly through the mitochondrial apoptotic pathway.

Obovatol regulates the mitogen-activated protein kinase (MAPK) pathway and suppresses the expression of MLL target genes. Studies have shown that the phosphorylation of the MAPK pathway can positively or negatively regulate cell mitosis, proliferation and apoptosis. In order to determine the effects of obovatol on the activation of Akt and the MAPK pathway, we evaluated the phosphorylation of Akt, JNK, ERK1/2 and p38 in obovatol-treated MM6 cells. We found that the total protein expression level of Akt was sustained, but the phosphorylation of Akt decreased significantly after $6 \mathrm{~h}$. The expression levels of p-JNK, p-ERK and p-p38 proteins following treatment with DMSO and obovatol for $6 \mathrm{~h}$ were very low, but increased significantly following treatment with obovatol $(60 \mu \mathrm{M})$ for $48 \mathrm{~h}$ (Fig. 3C). These findings suggest that obovatol activates the phosphorylation of JNK, p38 and ERK proteins to induce apoptosis in MM6 cells.

A recent study demonstrated that IKK/NF- $\kappa B$ signaling is a crucial factor in regulating the expression of MLL target genes and to maintain stem cell ability in MLL-rearranged leukemia (19). As MM6 is a leukemia cell line harboring the MLL-AF9 translocation, we investigated whether the expression levels of NF- $\mathrm{BB}$-associated proteins were affected by obovatol treatment. Treatment with obovatol decreased the protein expression levels of total IKK $\beta$ in a time-dependent manner (Fig. 3D) and significantly inhibited the phosphorylation of I $\mathrm{B} \alpha$ and $\mathrm{p} 65$, following the increased expression of 


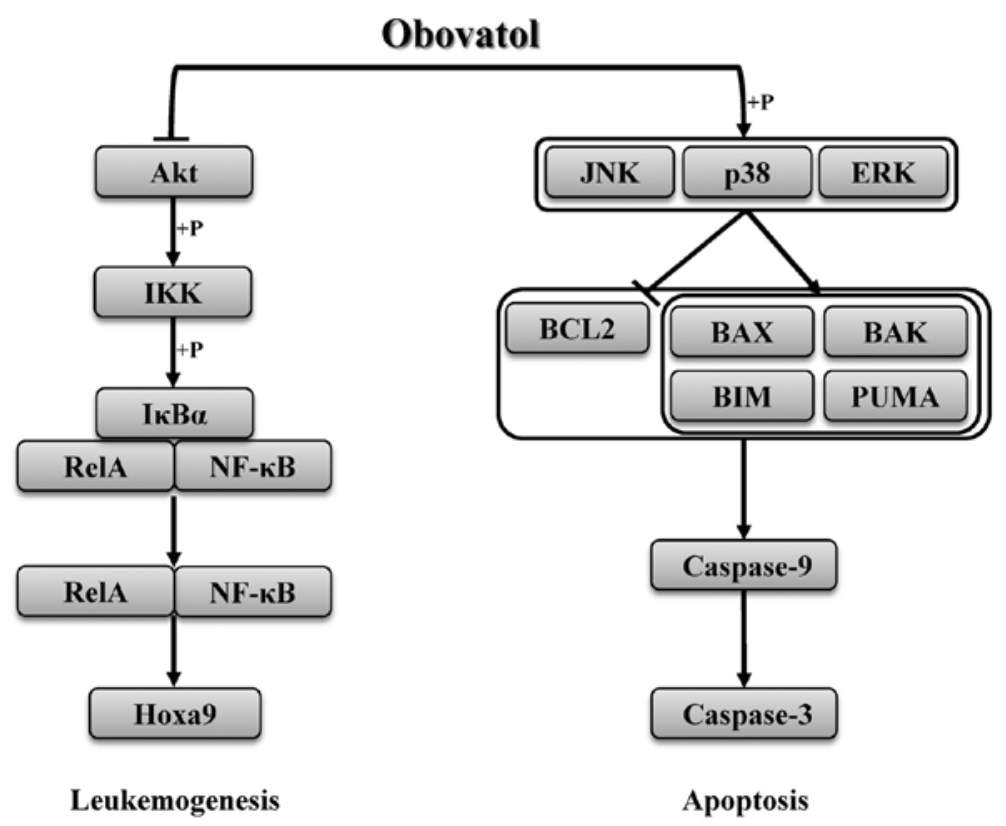

Figure 4. Schematic diagram illustrating the effects of obovatol on the induction of apoptosis of leukemia cells and the suppression of leukemogenesis.

total $\mathrm{I} \kappa \mathrm{B} \alpha$ (Fig. 3D). In addition, the protein expression of HOXA9, a MLL target gene, decreased due to its dependency on the activity of p-p65. These results suggest that obovatol suppresses the activity of the $\mathrm{NF}-\kappa \mathrm{B}$ signaling pathway and the expression of MLL target genes by hindering the phosphorylation of $\mathrm{I} \kappa \mathrm{B} \alpha$.

\section{Discussion}

Leukemia signifies a pathological condition characterized by the dysplasia of hematopoietic tissues, often with evidence of widespread metastases and tumors in distant organs, in addition to the striking feature of leukocytosis of immature cells in the peripheral blood (20). There is no single known cause for all the different types of leukemia. The few known causes, which are generally factors outside the control of the average individual, account for relatively few cases.

Several drugs have been developed for the treatment of leukemia, such as imatinib, nilotinib and dasatinib; however, there is no optimized treatment available to date for AML. Thus, the induction of apoptosis in leukemia cells may be a possible therapeutic strategy for AML. Numerous studies have suggested that obovatol induces apoptosis in prostate and colon cancer cells and thus, it may also be capable of inducing apoptosis in leukemia cells $(11-15,18)$. Based on these data, in this study, we evaluated the effects of obovatol on various leukemia cells and selected the MM6 cells for further analysis. MM6 cells have a MLL-AF9 fusion gene, which can affect the inhibition of proliferation. Based on the cell cycle analysis and PI/FITC dual staining results, we confirmed that obovatol inhibited cell growth and induced apoptosis in MM6 cells (Fig. 1). In addition, we observed an increase in activated caspase-3, caspase-9, and the expression of pro-apoptotic proteins (Bax, Bak, Bim and Puma) and a decrease in the expression of anti-apoptotic proteins (Bcl-2) (Fig. 2). The Bcl-2 family of proteins plays an important role regulating apoptosis. The Bcl-2 proteins reside at a critical point upstream of cellular damage and modulate apoptosis through the regulation of mitochondrial pathways (21). These results are in accordance with those of previous studies $(10,14)$ and suggest that obovatol induces apoptosis in leukemia cells through the mitochondrial apoptotic pathway (Fig. 4).

There are three major MAPK families, the ERK family, the JNK family and the p38 MAPK family. The MAPK signaling pathway is important for cell growth, proliferation and metabolism (22). Thus, studies on the function of the MAPK pathway in cancer cells continue to confirm the effects of obovatol as an anticancer agent $(15,22)$. Our results indicated that the increase in the phosphorylation of ERK, p38 and JNK, depending on the length of obovatol treatment, resulted in apoptosis upstream of anti- and pro-apoptotic genes (Fig. 3C). In addition, obovatol is known to suppress the NF- $\kappa \mathrm{B}$ signaling pathway in solid cancers (15). The phosphorylation of Akt triggers the activation of the $\mathrm{NF}-\kappa \mathrm{B}$ signaling through activated IKK $\alpha / \beta$ (23). The crucial target genes (HOXA9 and MEIS1) in leukemia stem cells and the maintenance of histone modifications are regulated by the NF- $\mathrm{BB}$ subunit, RelA (p65) in MLL leukemia (19). We evaluated the possibility that obovatol acts as an anti-leukemic agent by suppressing the activation of the $\mathrm{NF}-\kappa \mathrm{B}$ signaling pathway. Treatment with obovatol inhibited not only the expression of IKK $\beta$, but also the phosphorylation of Akt, I $\kappa \mathrm{B} \alpha$ and p65 (Fig. 3C and D). Additionally, HOXA9 protein expression was significantly diminished depending on the concentration of obovatol.

In conclusion, our data confirm that obovatol inhibits cell growth, induces apoptosis by regulating the MAPK signaling pathway, and suppresses the expression of MLL target genes by decreasing the phosphorylation of NF- $\kappa \mathrm{B}$ signaling-associated proteins (Fig. 4). The present study may aid in better understanding the effects of obovatol in MLL leukemia and may lead to the development of novel therapeutic approaches using obovatol as an anti-leukemic agent. 


\section{Acknowledgements}

This study was supported by a Korea Science and Engineering Foundation (KOSEF) grant funded by the government of Korea (MEST) (2011-0011163).

\section{References}

1. Teng CL, Yu CT, Hwang WL, et al: Effector mechanisms of sunitinib-induced G1 cell cycle arrest, differentiation, and apoptosis in human acute myeloid leukaemia HL60 and KG-1 cells. Ann Hematol 92: 301-313, 2013.

2. Nagase H, Ikeda K and Sakai Y: Inhibitory effect of magnolol and honokiol from Magnolia obovata on human fibrosarcoma HT-1080. Invasiveness in vitro. Planta Med 67: 705-708, 2001.

3. Park J, Lee J, Jung E, et al: In vitro antibacterial and antiinflammatory effects of honokiol and magnolol against Propionibacterium sp. Eur J Pharmacol 496: 189-195, 2004.

4. Wang T, Chen F, Chen Z, et al: Honokiol induces apoptosis through p53-independent pathway in human colorectal cell line RKO. World J Gastroenterol 10: 2205-2208, 2004.

5. Wang X, Wang Y, Geng Y, Li F and Zheng C: Isolation and purification of honokiol and magnolol from cortex Magnoliae officinalis by high-speed counter-current chromatography. J Chromatogr A 1036: 171-175, 2004.

6. Chang B, Lee Y, Ku Y, Bae K and Chung C: Antimicrobial activity of magnolol and honokiol against periodontopathic microorganisms. Planta Med 64: 367-369, 1998.

7. Ho KY, Tsai CC, Chen CP, Huang JS and Lin CC: Antimicrobial activity of honokiol and magnolol isolated from Magnolia officinalis. Phytother Res 15: 139-141, 2001.

8. Palayoor ST, Youmell MY, Calderwood SK, Coleman CN and Price BD: Constitutive activation of IkappaB kinase alpha and NF-kappaB in prostate cancer cells is inhibited by ibuprofen. Oncogene 18: 7389-7394, 1999.

9. Ikeda K, Sakai Y and Nagase H: Inhibitory effect of magnolol on tumour metastasis in mice. Phytother Res 17: 933-937, 2003.

10. Yang SE, Hsieh MT, Tsai TH and Hsu SL: Down-modulation of Bcl-XL, release of cytochrome $\mathrm{c}$ and sequential activation of caspases during honokiol-induced apoptosis in human squamous lung cancer $\mathrm{CH} 27$ cells. Biochem Pharmacol 63: 1641-1651, 2002.
11. Battle TE, Arbiser J and Frank DA: The natural product honokiol induces caspase-dependent apoptosis in B-cell chronic lymphocytic leukemia (B-CLL) cells. Blood 106: 690-697, 2005.

12. Hibasami H, Achiwa Y, Katsuzaki H, et al: Honokiol induces apoptosis in human lymphoid leukemia Molt 4B cells. Int J Mol Med 2: 671-673, 1998.

13. Hirano T, Gotoh M and Oka K: Natural flavonoids and lignans are potent cytostatic agents against human leukemic HL-60 cells. Life Sci 55: 1061-1069, 1994.

14. Ishitsuka K, Hideshima T, Hamasaki M, et al: Honokiol overcomes conventional drug resistance in human multiple myeloma by induction of caspase-dependent and -independent apoptosis. Blood 106: 1794-1800, 2005.

15. Choi MS, Lee SH, Cho HS, et al: Inhibitory effect of obovatol on nitric oxide production and activation of NF-kappaB/MAP kinases in lipopolysaccharide-treated RAW 264.7cells. Eur J Pharmacol 556: 181-189, 2007.

16. Kelly PN and Strasser A: The role of Bcl-2 and its pro-survival relatives in tumourigenesis and cancer therapy. Cell Death Differ 18: 1414-1424, 2011.

17. Ulukaya E, Acilan C and Yilmaz Y: Apoptosis: why and how does it occur in biology? Cell Biochem Funct 29: 468-480, 2011.

18. Lee SY, Yuk DY, Song HS, et al: Growth inhibitory effects of obovatol through induction of apoptotic cell death in prostate and colon cancer by blocking of NF-kappaB. Eur J Pharmacol 582: 17-25, 2008.

19. Kuo HP, Wang Z, Lee DF, et al: Epigenetic roles of MLL oncoproteins are dependent on NF-кB. Cancer cell 24: 423-437, 2013.

20. Lucia SP: Leukemia: Evaluation of the Therapy. Cal West Med 55: 119-123, 1941.

21. Cory S, Huang DC and Adams JM: The Bcl-2 family: roles in cell survival and oncogenesis. Oncogene 22: 8590-8607, 2003.

22. Pearson G, Robinson F, Beers Gibson T, et al: Mitogen-activated protein (MAP) kinase pathways: regulation and physiological functions. Endocr Rev 22: 153-183, 2001.

23. Kane LP, Shapiro VS, Stokoe D and Weiss A: Induction of NF-kappaB by the Akt/PKB kinase. Curr Biol 9: 601-604, 1999. 